\title{
Symmetry of Surface States in Graphene Superlattices Terminated With Magnetic Cap Layers
}

HuaizheXu ( $\sim$ hzxu@buaa.edu.cn )

Beihang University https://orcid.org/0000-0003-1416-9360

Qiqi Yan

Beihang University

Liying Wang

Beihang University

Sansheng Wang

Beihang University

Yaping Zhang

University of Nottingham - Ningbo China

Research Article

Keywords: Surface States, Symmetry, Superlattice of Complex Basis, Single layer Graphene

Posted Date: March 10th, 2021

DOI: https://doi.org/10.21203/rs.3.rs-229560/v1

License: (c) (1) This work is licensed under a Creative Commons Attribution 4.0 International License. Read Full License 


\title{
Symmetry of Surface States in Graphene Superlattices Terminated with Magnetic Cap Layers
}

\author{
H. Z. Xu ${ }^{1, *}$, Q. Q. Yan ${ }^{1}$, L. Y. Wang ${ }^{1}$, S. S. Wang ${ }^{1}$, and Y. P. Zhang ${ }^{2, * *}$ \\ ${ }^{1}$ State Key Laboratory of Software Development Environment and Department of Physics, Beihang \\ University, Beijing100191, China \\ ${ }^{2}$ Faculty of Engineering, University of Nottingham Ningbo China, Ningbo 315100, China
}

\begin{abstract}
The symmetry of surface states in graphene superlattices (SLs) terminated with a magnetic cap layer has been identified with numerical calculations. It is found that the surface states in pure electric SLs with $A_{s} \geq 0$ are symmetric about $k_{y}=0$ to those with $A_{s} \leq 0$. While those in pure magnetic SLs with the same $A_{s}$ are symmetric about $E=0$. Additionally, the surface states in SLs of general complex electric/magnetic basis show no symmetry. The symmetry of surface states in terminated SLs is mainly determined by the nature of the basis and its configuration. These interesting results provide a useful guideline to the experimental exploration of carbon-based quantum electronic and optoelectronic devices.
\end{abstract}

PACS numbers: 73.21.Cd, 73.20.At, 75.70.Cn.

Keywords: Surface States; Symmetry; Superlattice of Complex Basis; Single layer Graphene.

*Corresponding author, e-mail address: hzxu@,buaa.edu.cnl;

** Yaping.ZHANG@nottingham.edu.cn 


\section{Introduction}

The complex-basis SLs normally consist of three or four different layers instead of two, such as a stepwell basis and a $\delta$-doped SLs, and the basis of biperiodic SLs consist of two-wells and two-barriers. ${ }^{[1-}$ ${ }^{16]}$ In practical applications, SLs are normally finite in length and terminated on one end or both ends, the termination interrupts the periodicity of infinite SL and introduces an asymmetric perturbation potential to the SL, which leads to the appearance of surface states within the energy band gap. The surface states are important for optoelectronic characteristics of SLs. As the properties of the surface states depend on the configurations and the sequence of the complex-basis SL basis, the complex-basis SL may provide a greater degree of freedom to influence the behavior of surface states through various terminating configurations of SL. ${ }^{[17-23]}$

Graphene is a monolayer of carbon atoms arranged in a honeycomb pattern, and it has attracted tremendous attention since 2004. ${ }^{[24,25]}$ It is even considered as a promising material of next-generation carbon-based electronics to replace silicon in the near future, due to Graphene's unique electronic properties. The electron transport in graphene is described by a massless Dirac equation, rather than the Schrödinger equation. Up to now, little research work has been carried out on the surface states in graphene SLs, let alone the influence of different kinds of cap layer on the surface states. Previously, Jiang et al. ${ }^{[26]}$ studied Tamm surface states between a graphene SL and a homogeneous electric potential barrier, they observed the backward electronic Tamm states in graphene SL. Pekh and Silin ${ }^{[27]}$ calculated the Tamm minibands in graphene-based planar superlattices. Lin et. al. ${ }^{[28,29]}$ demonstrated that some special complex basis SLs could generate an energy bandgap of suitable width in the gapless pristine graphene. Recently, Wang et. al. ${ }^{[30]}$ have investigated the surface states in three types of SLs terminated with electric cap layers, and they have discovered some symmetrical properties of the surface states in those SLs. However, the symmetry of surface states in graphene SLs terminated with magnetic cap layer, and the influence of the cap layer on the surface states symmetry, have not yet been investigated.

In this paper, we will numerically calculate the distributions of surface states in semi-infinite SLs of three types of complex basis terminated with magnetic vector potentials, and analyze the relationship of surface states symmetrical characteristics with the basis of the underlying SL, including the potential and configuration. The symmetrical properties of surface states are expected to be very helpful in the 
experimental exploration of carbon-based quantum electronic and optoelectronic devices.

\section{Theoretical model}

Three types of complex basis are considered, and the basis of the SLs are assumed to consist of fourlayers of two anti-parallel potentials and two anti-parallel magnetic potentials. In order to avoid the possible confusion resulting from a shift of the SL band structure, we also assume the basis of SLs are of zero average electric potential and zero average magnetic field. The SL is infinite into $\mathrm{x}>0$, and is terminated by a homogeneous magnetic vector potential at $\mathrm{x}=0$. The alignment of a model four-layer basis SL and their potential configurations, as well as the termination potential, are schematically depicted in Fig. 1.

The magnetic vector potentials of cap layer and SL, as well as the electric potential $\mathrm{U}(x)$ in $\mathrm{SL}$ are illustrated as:

$$
\begin{aligned}
& \stackrel{\rho}{A}(x)=\left\{\begin{array}{cc}
A_{s} y & x<0 \\
A_{d}[\Theta(x-d)-\Theta(x-d-b)-\Theta(x-2 d-b)+\Theta(x-2 d-2 b)] & x>0
\end{array}\right\}, \\
& U(x)=\left\{\begin{array}{cc}
0 & x<0 \\
U[\Theta(x)-\Theta(x-d)-\Theta(x-d-b)-\Theta(x-2 d-b)] & x>0
\end{array}\right\},
\end{aligned}
$$

The electron transport in graphene is described by the Dirac equation:

$$
H=v_{F} \rho \cdot(\not{P}+e \stackrel{\mu}{\rho}(x))+e U(x),
$$

where $v_{F} \approx 10^{6} \mathrm{~m} / \mathrm{s}$ is the Fermi velocity, $e$ is the absolute value of the electron charge, $\stackrel{p}{P}$ is the momentum operator, and $\stackrel{\mu}{\sigma}=\left(\sigma_{x}, \sigma_{y}\right)$ is the $2 \times 2$ Pauli matrix.

Following the procedure of Ref.[30], we derive the surface state condition by using the transfer matrix method within the envelope function approximation. ${ }^{[28-32]}$ The wave function continuity condition at interface $\mathrm{x}=0$ is expressed as:

$$
\left(\begin{array}{c}
\psi_{A S}(x) \\
\psi_{B S}(x)
\end{array}\right)=\left(\begin{array}{c}
1 \\
\gamma_{S}
\end{array}\right) a_{S} e^{q_{S} x},
$$


where $\gamma_{s}=i E /\left(q_{s}+k_{y}+A_{s}\right), \quad q_{s}=\left[\left(k_{y}+A_{s}\right)^{2}-E^{2}\right]^{1 / 2}$ is the $x$-component of the wave vector and $a_{s}$ is the amplitude of wave function in the cap layer. Again, all quantities are expressed in dimensionless units, i.e. $l \rightarrow l_{0} l, k \rightarrow k_{0} k, E \rightarrow E_{0} E, A \rightarrow B_{0} l_{0} A$, where $k_{0}=1 / l_{0}, E_{0}=\eta v_{F} / l_{0}, \quad B_{0}=\eta / e l_{0}^{2}$. For a realistic value $B_{0}=0.1 \mathrm{~T}, l_{0}=811 \AA$ and $E_{0}=7.0 \mathrm{meV}$.

The energy band edges of the infinite SL satisfy the following expression:

$\left|M_{11}+M_{22}\right| / 2=1$

Here, $M_{i, j}(i, j=1,2)$ are the elements of total matrix for the basis of SL. ${ }^{[28-32]}$

$M=\left(\begin{array}{ll}M_{11} & M_{12} \\ M_{21} & M_{22}\end{array}\right)=S_{U+} S_{A+} S_{U-} S_{A-}$,

with

$S_{U \pm}=\left(\begin{array}{cc}\operatorname{Cos}\left(k_{ \pm} b\right)-\frac{k_{y}}{k_{ \pm}} \operatorname{Sin}\left(k_{ \pm} b\right) & -i \frac{(E \mu U)}{k_{ \pm}} \operatorname{Sin}\left(k_{ \pm} b\right) \\ -i \frac{(E \mu U)}{k_{ \pm}} \operatorname{Sin}\left(k_{ \pm} b\right) & \operatorname{Cos}\left(k_{ \pm} b\right)+\frac{k_{y}}{k_{ \pm}} \operatorname{Sin}\left(k_{ \pm} b\right)\end{array}\right)$,

$S_{A \pm}=\left(\begin{array}{cc}\operatorname{Cos}\left(K_{ \pm} d\right)-\frac{\left(k_{y} \pm A\right)}{K_{ \pm}} \operatorname{Sin}\left(K_{ \pm} d\right) & -i \frac{E}{K_{ \pm}} \operatorname{Sin}\left(K_{ \pm} d\right) \\ -i \frac{E}{K_{ \pm}} \operatorname{Sin}\left(K_{ \pm} d\right) & \operatorname{Cos}\left(K_{ \pm} d\right)+\frac{\left(k_{y} \pm A\right)}{K_{ \pm}} \operatorname{Sin}\left(K_{A \pm} d\right)\end{array}\right)$

Here, $k_{0}=\sqrt{E^{2}-k_{y}^{2}}, k_{ \pm}=\sqrt{(E \mu U)^{2}-k_{y}^{2}}, K_{ \pm}=\sqrt{E^{2}-\left(k_{y}^{2} \pm A\right)}$, with (+) for electric (magnetic) potential barrier and (-) for electric (magnetic) potential well.

The surface states at the point $x=0$ is given as:

$\frac{i E}{q_{s}+k_{y}+A_{s}}=\frac{M_{21}}{M_{11}-e^{i K l}}$

For the existence of surface states two conditions must be satisfied, $q_{x}$ should be real and $q_{x}>0$; and the Bloch wave number $K$ should be complex. 
$K=\frac{n \pi}{d}+i q_{s}\left(q_{s}>0, n=0,1,2,3 \ldots\right)$

Here we have only given a short derivation process for brevity and completeness, for more details please refer to Ref. [30]. It is obvious that termination of SLs with a magnetic cap layer could have a very important influence on the surface states locations and their dispersion relation. Thus, the cap layer could be a useful technique for modulating the surface states of the SLs.

In the following section, we will numerically calculate the surface states with Eqn. (9) in semi-infinite SLs with three-typical complex basis terminated with a magnetic cap layer of different magnetic vector potentials, and analyze the surface states symmetric properties, as well as its relation to the nature of basis and its configuration of SLs. The energy bands for an infinite SL calculated with Eqn.(5) are depicted by the gray shaded regions for reference.

\section{Results and discussions}

Fig. 2 shows the energy bands of an infinite SL and the surface states in terminated SLs of pure electric basis, with the cap layer of seven magnetic potentials $A_{s}\left(A_{s}=0, \pm 1, \pm 2\right)$, respectively. The other parameters are fixed at $b=d=0.5, U=3$ and $A_{d}=0$. The surface states are existing in the forbidden minigaps and they are illustrated with color curves. The energy bands of infinite SL are symmetry for both $k_{y}=0$ and $E=0$, when the two electric potentials are arranged as antiparallel in a basis of pure SL with $\langle U>=0$, and there is no bandgap or minigap appearance at the Dirac point $k_{y}=0$. Without a magnetic cap layer (with $A_{s}=0$ ), the surface states in semi-infinite SL are situated at the bottom of the first electron minigap, or at the top of hole minibands, and the surface state versus $k_{y}$ is symmetric about $k_{y}=0$. With a cap layer of magnetic vector $A_{s}>0$, the surface states in terminated SLs appear mainly as hole states with $k_{y}<0$, or as electron states with $k_{y}>0$. With a cap layer of magnetic vector $A_{s}<0$, the surface hole states appear mainly at $k_{y}>0$, or as electron states at $k_{y}<0$. Although the surface states in SL shift entirely to $k_{y}>0$ (or $k_{y}<0$ ), when it is terminated 
with a cap layer of $A_{s}>0$ ( or $A_{s}<0$ ), the surface states versus $k_{y}$ with $A_{s}<0$ are symmetric about $k_{y}=0$ to those with $A_{s}>0$.

Fig.3 shows the energy bands of an infinite SL and the surface state in terminated SLs of a pure magnetic basis, and the cap layer is assumed to be of seven magnetic potentials $A_{s}\left(A_{s}=0, \pm 1, \pm 2\right)$, respectively. The other parameters are taken as $b=d=0.5, A_{d}=3$ and $U=0$. The infinite SL's energy bands are symmetry at both $k_{y}=0$ and $E=0$, when the two magnetic vector potentials are chosen as antiparallel in a basis of pure magnetic SL with $\left\langle A_{d}\right\rangle=0$. There is no bandgap Dirac point, but some new minigaps appear at $k_{y}=0$. The surface states in a pure magnetic semi-infinite SL without cap layer (i.e. with $A_{s}=0$ ) appear at both conduction and valence gaps with same $k_{y}$, and the surface state versus $k_{y}$ is symmetric about $E=0$. When terminated with a cap layer of magnetic vector potential $A_{s}>0$, the surface states in the terminated SL appear mainly at $k_{y}>0$, while the surface states are mainly appear at $k_{y}<0$ when terminated with a cap layer of magnetic vector potential $A_{s}<0$. Different from the pure electric SLs, the surface states in pure magnetic SLs are symmetric about $E=0$, no matter the value of $A_{s}$.

Fig.4 shows the energy bands of an infinite SL and the surface states in terminated SLs of a complex electric/magnetic basis, and the cap layer is assumed to be of seven magnetic potentials $A_{s}\left(A_{s}=0\right.$, $\pm 1, \pm 2$ ), respectively. The other parameters are taken as $b=d=0.5, A_{d}=3$ and $U=3$. When combining the pure SL of Fig. 2 with the pure magnetic SLs of Fig.3 together to make a complex electric/magnetic basis SLs of $\langle U\rangle=0$ and $\left\langle A_{d}\right\rangle=0$, the infinite SL's energy bands are still of symmetry about $k_{y}=0$ and $E=0$. In addition, a moderate energy bandgap is generated at the Dirac point, and some minigaps also appear at $k_{y}=0 .{ }^{[28]}$ Surprisingly, the surface states in SLs of a general complex electric/magnetic basis display no symmetry, no matter how the SL is terminated with or without a magnetic cap layer. 
Although the surface states locations and their dispersion relations with $k_{y}$ could be completely different from each other when varying the cap layer types and/or the termination potential strengths, the symmetry of surface states in SLs is never disturbed. The symmetry of surface states is always kept unchanged for SLs of identical basis and configuration, no matter whether the SLs are free of a cap layer, terminated with an electric or a magnetic cap layer. These interesting results indicate that the symmetry of surface states in semi-infinite SLs is mainly determined by their basis and configuration, without any influence from termination types (electric or magnetic) or their values (positive, zero or negative).

\section{Conclusion}

The symmetry of the surface states in magnetically terminated SLs of three types of basis are clearly identified with the help of numerical calculations. The surface states with $A_{s} \geq 0$ show symmetry to those with $A_{s} \leq 0$ about $k_{y}=0$ in terminated SLs with a pure electric and antiparallel basis of $<U>=0$, and with the same absolute value $\left|A_{s}\right|$. The surface states in terminated SLs at the same $A_{s}$ are all of symmetry to $E=0$ in SLs with a pure magnetic and antiparallel basis of $\left\langle A_{d}\right\rangle=0$, For SLs with a complex electric/magnetic basis of $\langle U\rangle=0$ and $\left\langle A_{d}\right\rangle=0$, the surface states exhibit no symmetry about $k_{y}=0$ or $E=0$. It is also found that the symmetry of surface states in SLs is mainly determined by the nature of the basis and its configuration of SLs, irrespective to the termination potential types (magnetic or electric) nor their values (positive, zero or negative). These interesting results provide an important information for experimental researchers to work on graphene-based potential device application.

Acknowledgements: This work was supported by the State Key Laboratory of Software Development Environment (Grant No. SKLSDE-2020ZX-30). 


\section{References}

[1] P. F. Yuh, K. L. Wang, "Formalism of the Kronig-Penney model for superlattices of variable basis", Phys. Rev. B38, 13307-13315(1988).

[2] W. Bloss, "Surface states of a semi-infinite superlattice”, Phys. Rev. B44, 8035(1991).

[3] S. G. Tikhodeev, “Tamm minibands in superlattices”, Solid State Commun., 78, 339-342(1991).

[4] H. Ohno, E. E. Mendez, J. A. Brum, J. M. Hong, F. Agullo-Rueda, L. L. Chang, L. Esaki, “Observation of "Tamm states" in superlattices", Phys. Rev. Lett., 64, 2555-2558(1990).

[5] H. Ohno, E. E. Mendez, A. Alexandrou, and J. M. Hong, "Tamm states in superlattices", Surf. Sci., 267, 161-165(1992).

[6] F. Y. Huang and H. Morkoç, "Electronic surface state (Tamm state) under electric field in semiconductor superlattices", J. Appl. Phys. 71, 524-526(1992);

[7] G. Ihm, S. K. Noh, M. L. Falk, and K. Y. Lim, "Interface localized states in coupled superlattices", J. Appl. Phys., 72, 5325-5328(1992).

[8] G. Ihm, M. L. Falk, S. K. Noh, and S. J. Lee, "Symmetry-dependent localization in a finite superlattice”, Phys. Rev. B46, 9564-9568(1992).

[9] J. Arriaga, F. García-Moliner, and V. R. Velasco, "Surface States in semiinfinite superlattices”, Prog. Surf. Sci., 42, 271-279(1993).

[10] W. Glessner and R. H. Yu, "Novel surface electronic states in finite superlattices", J. Appl. Phys. 73, 4070-4071(1993);

[11] N. A. Gippius, G. A. Kopelevich, A. Yu. Sivachenko, S. G. Tikhodeev and A. L. Yablonskii, “Interface (Tamm) minibands in superlattices", Surf. Sci. Lett. 264, L223-L226(1992).

[12] R. H. Yu, "Tamm states in finite semiconductor superlattices: Influence of accumulation and depletion layers", Phys. Rev. B47,1379-1382 (1993).

[13] A. A. Chowdhury and C. M. Maziar, "Modeling of Tamm like states in superlattices from a simple tight-binding method", J. Appl. Phys., 76, 3902-3904(1994). 
[14] M. Steslicka, “Tamm surface states in semiconductor superlattices”, Prog. Surf. Sci., 50, 6576(1995).

[15] A. Akjouj, and B. Djafari-Rouhani, "Novel surface states in lateral magnetic superlattices", Solid State Commun. 103, 161-165(1997).

[16] R. Kucharczyk, B. Brzostowski, B. Djafari-Rouhani, "Density of states of superlattices with multiple layers per period", Physica E5, 280-290(2000).

[17] R. Kucharczyk, M. Steslicka, B. Djafari-Rouhani, "Electronic level structure and density of states of a terminated biperiodic superlattice”, Phys. Rev. B62, 4549-4556(2000).

[18] R. Kucharczyk, M. Steslicka, B. Djafari-Rouhani, "Surface states in double-well superlattices: symmetric vs asymmetric interwell coupling”, Surf. Sci., 482-485, 648-653(2001).

[19] M. Steslicka, R. Kucharczyk, A. Akjouj, B. Djafari-Rouhani, L. Dobrzynski, S. G. Davison, “Localised electronic states in semiconductor superlattices”, Surf. Sci. Reports, 47, 93-196(2002).

[20] J. Kłos and H. Puszkarski, "Conditions of coexistence of Tamm and Shockley states in a superlattice with a perturbed surface”, Phys. Rev. B 68, 045316-8(2003).

[21] P. Pereyra, "Eigenvalues, eigenfunctions, and surface states in finite periodic systems", Annals of Physics 320 (2005) 1-20; (Erratum) ibid, 321, 1276 -1276(2006).

[22] W. J. Hsueh, H. C. Chen, "Calculation of electronic surface states in superlattices via graph formulations", Phys. Rev, E76, 057701-4(2007).4pp

[23] Y. O. Averkov, V. M. Yakovenko, "Effect of the $\delta$-shaped quantum well at the one-dimensional lattice boundary on properties of Tamm type surface electronic states", Physics of Solid. States, $54,630-635(2012)$.

[24] K. S. Novoselov, A. K. Geim, S. V. Morozov, D. Jiang, Y. Zhang, S. V. Dubonos, I. V. Grigorieva, A. A. Firsov, "Electric field effect in atomically thin carbon films", Science 306, 666-669 (2004).

[25] K. Geim, "Graphene: status and prospects", Science 324, 1530-1534(2009).

[26] H. T. Jiang, Z. L. Wang, Z. G. Wang, H. Chen, "Backward electronic Tamm states in graphenebased heterostructures”, Phys. Lett., A375, 1014-1018(2011). 
[27] Pavel Pekh, A. P. Silin, "Tamm minibands in graphene-based planar superlattices”, Physics of Wave Phenomena 25, 249-253(2017).

[28] X. Lin, H. L. Wang, H. Pan, H. Z. Xu, "Gap opening and tuning in single-layer graphene with combined electric and magnetic field modulation ”, Chin. Phys. B20, 047302-10(2011).

[29] X. Lin, H. L. Wang, H. Pan, H. Z. Xu, "Gap opening in single-layer graphene in the presence of periodic scalar and vector potentials within the continuum model", Phys. Lett. A376, 584589(2012).

[30] L. Y. Wang, Q. Q. Yan, H. Z. Xu, H. L. Wang, G. L. Zhang, X. P. Zhou, “Tamm states in graphenebased different combined magneto-electric superlattice heterostructures”, Phys. Lett., A380, 32973301(2016).

[31] H. Z. Xu, S. Feng, Y. P. Zhang, J. L. Wang, S. C. Zhang, "Resonant tunneling though an asymmetrical two magnetic-barrier structure on single layer graphene”, Opt. Quant. Electron, 49, 250-9(2017).

[32] H. Z. Xu, S. Feng, Y. P. Zhang, "Resonant peak splitting infinite periodic superlattices with an unit cell of two barriers and two wells on monolayer graphene”, Opt. Quant. Electron, 51, 158-15(2019).

\section{Figure captions}


Fig. 1. Schematic diagram for the model of a magnetic cap layer terminated graphene SL, the interface is assumed at $x=0$.

Fig. 2. The energy bands of infinite SL and surface states in pure electric SLs terminated with different magnetic cap layer of $A_{S}\left(A_{S}=-2,-1,0,1,2\right)$, respectively. The basis of pure electric SL is U$A_{d}-(-U)-\left(-A_{d}\right)$ with $b=d=0.5, U=3$, and $A_{d}=0$.

Fig. 3. The energy bands of infinite SL and surface states in pure magnetic SLs terminated with different magnetic cap layer of $A_{S}\left(A_{s}=-2,-1,0,1,2\right)$, respectively. The basis of pure magnetic SL is U-A $-(-U)-\left(-A_{d}\right)$ with $\quad b=d=0.5, U=0$, and $A_{d}=3$.

Fig. 4. The energy bands of infinite SL and surface states in complex electric/magnetic SLs terminated with different magnetic cap layer of $A_{s}\left(A_{s}=-2,-1,0,1,2\right)$, respectively. The basis of complex electric/magnetic SL is $U-A_{d}-(-U)-\left(-A_{d}\right)$ with $b=d=0.5, U=3$, and $A_{d}=3$. 


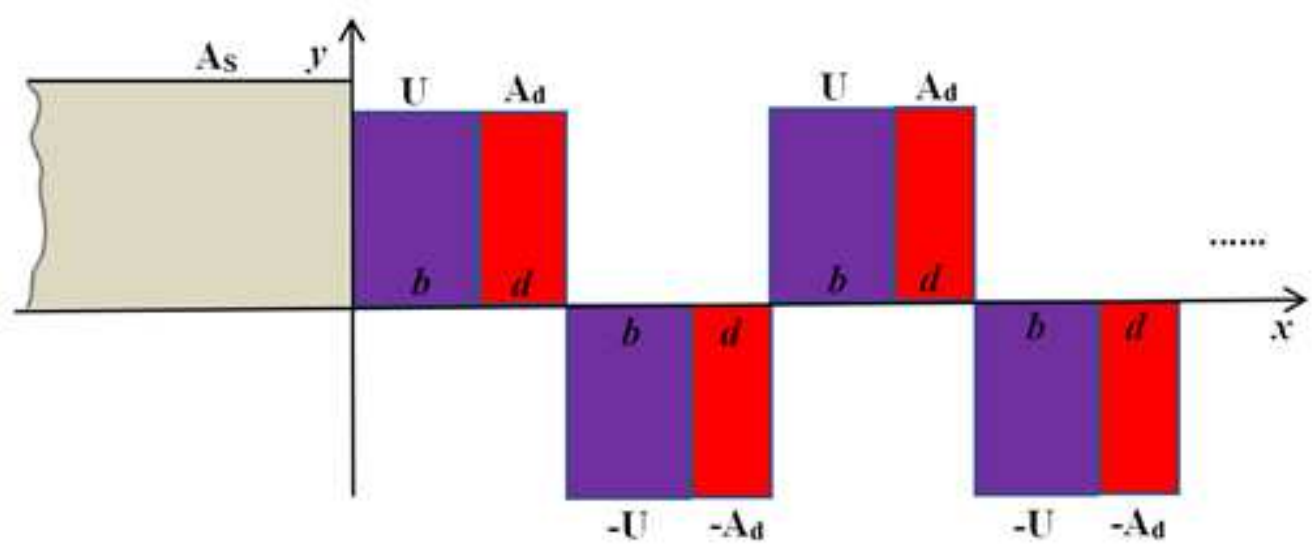

\section{Figure 1}

Schematic diagram for the model of a magnetic cap layer terminated graphene SL, the interface is assumed at $\mathrm{x}=0$.

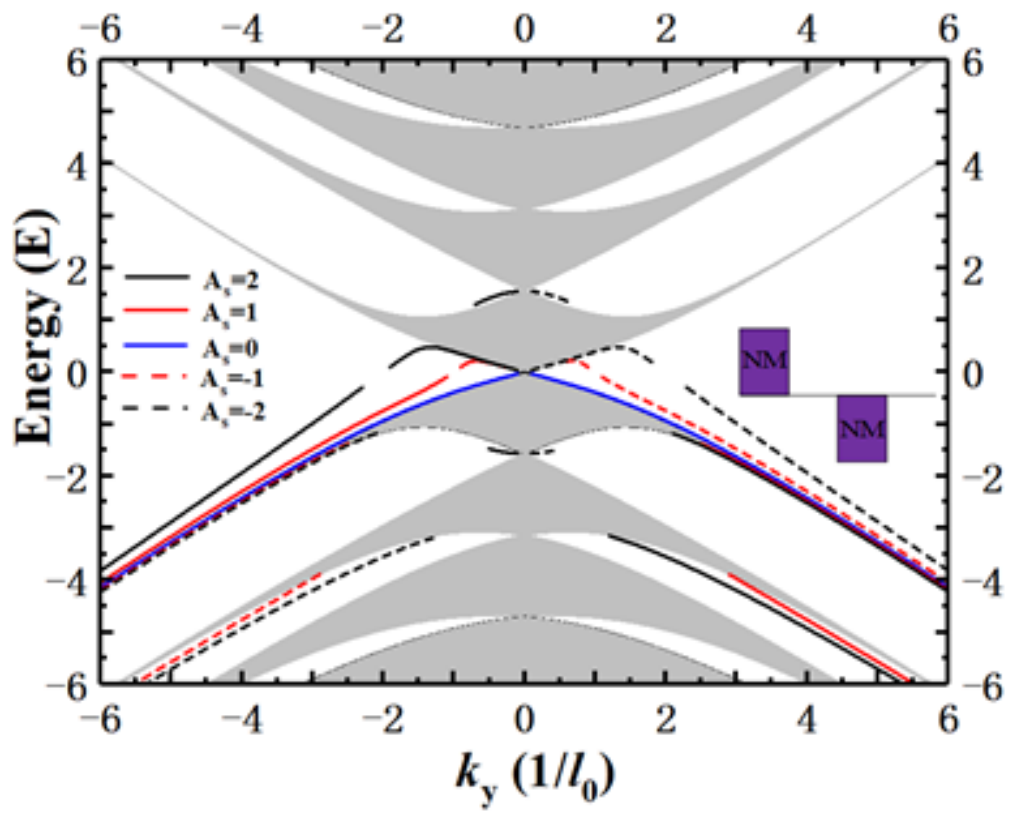

Figure 2

The energy bands of infinite SL and surface states in pure electric SLs terminated with different magnetic cap layer of As (As=-2, -1, 0, 1, 2), respectively. The basis of pure electric SL is U-Ad-(-U)-(-Ad) with $b=d=0.5, U=3$, and $A d=0$ 


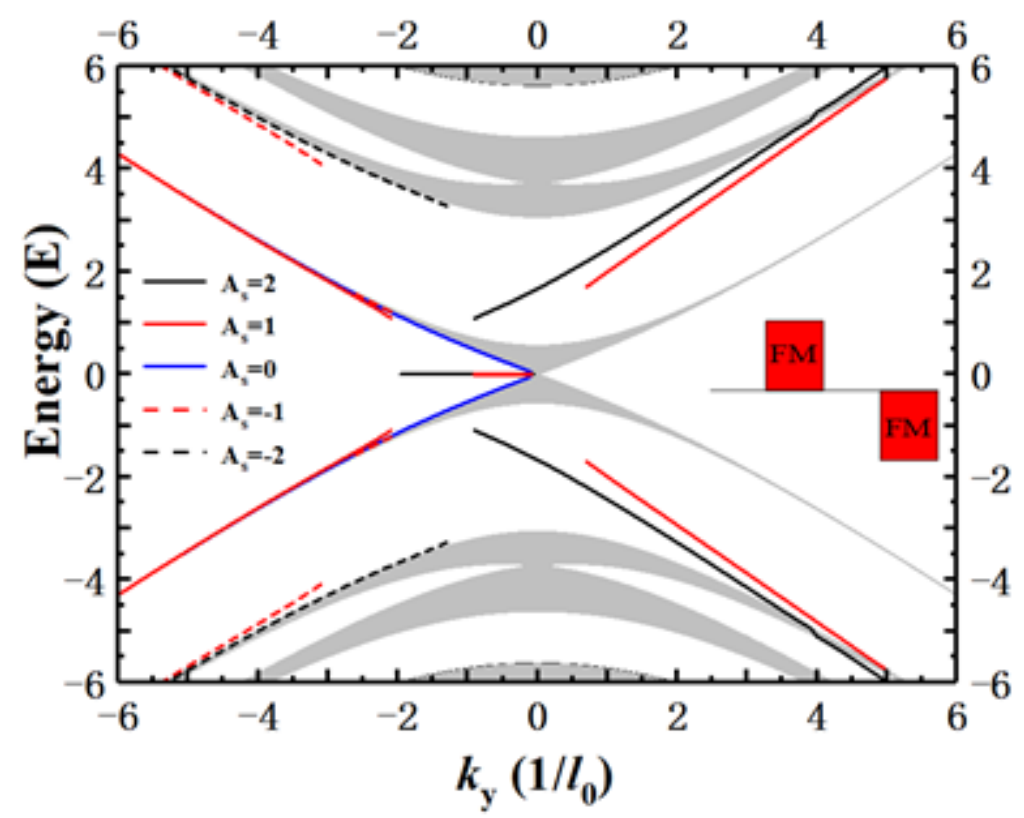

Figure 3

The energy bands of infinite $S L$ and surface states in pure magnetic SLs terminated with different magnetic cap layer of As $(A s=-2,-1,0,1,2)$, respectively. The basis of pure magnetic SL is U-Ad-(-U)-(-Ad) with $b=d=0.5, U=0$, and $A d=3$.

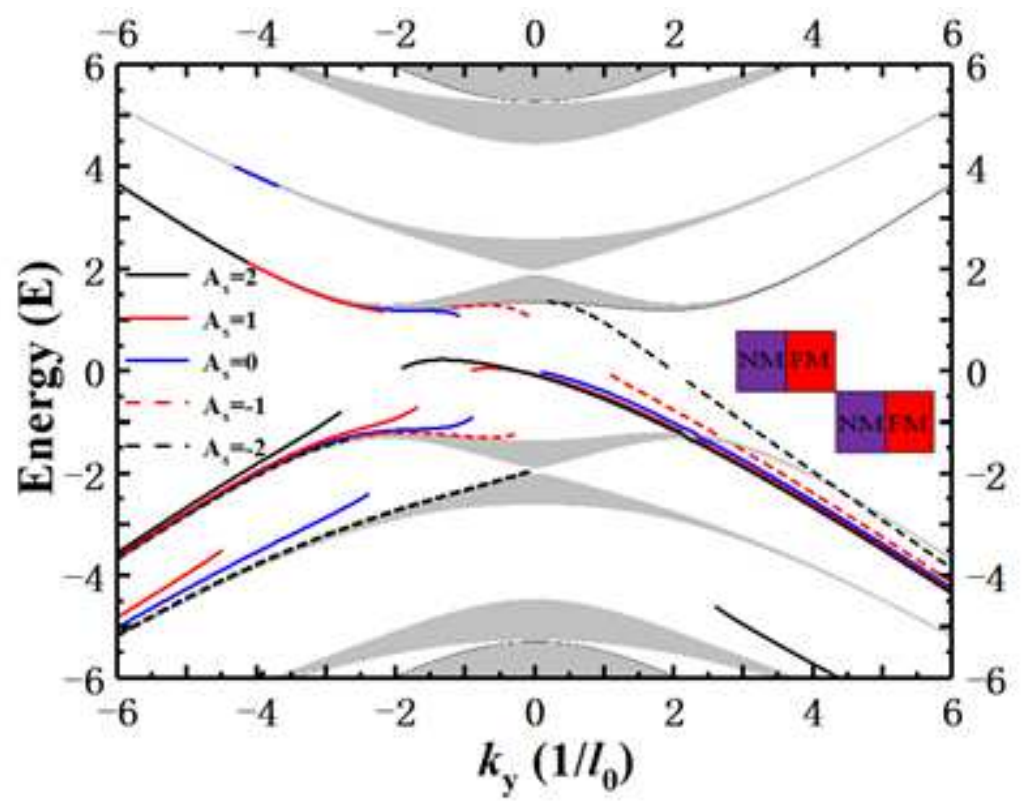

Figure 4

The energy bands of infinite SL and surface states in complex electric/magnetic SLs terminated with different magnetic cap layer of $A s(A s=-2,-1,0,1,2)$, respectively. The basis of complex electric/magnetic $S L$ is $U-A d-(-U)-(-A d)$ with $b=d=0.5, U=3$, and $A d=3$. 